International Journal of Pure and Applied Mathematics

Volume 102 No. 3 2015, 473-482

ISSN: 1311-8080 (printed version); ISSN: 1314-3395 (on-line version)

url: http://www.ijpam.eu

doi: http://dx.doi.org/10.12732/ijpam.v102i3.5

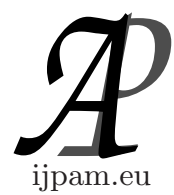

\title{
SUBCLASSES OF BI-UNIVALENT FUNCTIONS BASED ON HOHLOV OPERATOR
}

\author{
O.S. Babu ${ }^{1}$, C. Selvaraj ${ }^{2}$, G. Murugusundaramoorthy ${ }^{3}$ \\ ${ }^{1}$ Department of Mathematics \\ Dr. Ambedkar Govt. Arts College \\ Chennai, 600039, INDIA \\ ${ }^{2}$ Department of Mathematics \\ Presidency College (Autonomous) \\ Chennai, 600005, INDIA \\ ${ }^{3}$ School of Advanced Sciences \\ VIT University \\ Vellore, 632 014, INDIA
}

\begin{abstract}
In this paper, we introduce two new subclasses of the function class $\Sigma$ of bi-univalent functions defined in the open unit disc based on Hohlov Operator. Furthermore, we find estimates on the coefficients $\left|a_{2}\right|$ and $\left|a_{3}\right|$ for functions in these new subclasses. Also consequences of the results are pointed out.
\end{abstract}

AMS Subject Classification: 30C45

Key Words: analytic functions, Bi-convex functions, Bi-starlike functions, Bi-univalent functions, Hohlov operator, Univalent functions

Received: January 31, 2015

(C) 2015 Academic Publications, Ltd. url: www.acadpubl.eu

${ }^{\S}$ Correspondence author 


\section{Introduction and Definitions}

Let $\mathcal{A}$ denote the class of functions of the form

$$
f(z)=z+\sum_{n=2}^{\infty} a_{n} z^{n}
$$

which are analytic in the open unit disc $\mathbb{U}=\{z: z \in \mathbb{C}$ and $|z|<1\}$. Further, by $\mathcal{S}$ we shall denote the class of all functions in $\mathcal{A}$ which are univalent in $\mathbb{U}$.It is well known that every function $f \in \mathcal{S}$ has an inverse $f^{-1}$, defined by

$$
f^{-1}(f(z))=z, z \in \mathbb{U}
$$

and

$$
f\left(f^{-1}(w)\right)=w,|w|<r_{0}(f) ; r_{0}(f) \geq \frac{1}{4}
$$

where

$$
f^{-1}(w)=w-a_{2} w^{2}+\left(2 a_{2}^{2}-a_{3}\right) w^{3}-\left(5 a_{2}^{3}-5 a_{2} a_{3}+a_{4}\right) w^{4}+\ldots
$$

A function $f \in \mathcal{A}$ is said to be bi-univalent in $\mathbb{U}$ if both $f(z)$ and $f^{-1}(z)$ are univalent in $\mathbb{U}$.

In 1967, Lewin [5] investigated the bi-univalent function class $\Sigma$ and showed that $\left|a_{2}\right|<1.51$. Subsequently, Brannan and Clunie [1] conjectured that $\left|a_{2}\right| \leq$ $\sqrt{2}$. Netanyahu [7], on the other hand, showed that $\max _{f \in \Sigma}\left|a_{2}\right|=\frac{4}{3}$. The coefficient estimate problem for each of the following Taylor-Maclaurin coefficients $\left|a_{n}\right|$ for $n \in \mathbb{N} \backslash\{1,2\} ; \mathbb{N}:=\{1,2,3, \ldots\}$ is presumably still an open problem.

Brannan and Taha [2] introduced certain subclasses of the bi-univalent function class $\Sigma$. A function $f \in \mathcal{A}$ is in the class $\mathcal{S}_{\Sigma}^{*}(\alpha)$ of strongly bistarlike of order $\alpha(0 \leq \alpha<1)$, if each of the following condition is satisfied: $f \in \Sigma,\left|\arg \left(\frac{z f^{\prime}(z)}{f(z)}\right)\right|<\frac{\alpha \pi}{2}$, and $\left|\arg \left(\frac{w g^{\prime}(w)}{g(w)}\right)\right|<\frac{\alpha \pi}{2}, w \in \mathbb{U}$; and the class $\mathcal{K}_{\Sigma}^{*}(\alpha)$ strongly bi-convex of order $\alpha(0 \leq \alpha<1)$, if each of the following condition is satisfied: $f \in \Sigma,\left|\arg \left(1+\frac{z f^{\prime}(z)}{f^{\prime}(z)}\right)\right|<\frac{\alpha \pi}{2}$, and $\left|\arg \left(1+\frac{w g^{\prime \prime}(w)}{g^{\prime}(w)}\right)\right|<$ $\frac{\alpha \pi}{2}, \quad w \in \mathbb{U}$; where the function $g$ is given

$$
g(w)=w-a_{2} w^{2}+\left(2 a_{2}^{2}-a_{3}\right) w^{3}-\left(5 a_{2}^{3}-5 a_{2} a_{3}+a_{4}\right) w^{4}+\ldots
$$

and $g$ is the extension of $f^{-1}$ to $\mathbb{U}$ and found non-sharp estimates on the first two Taylor-Maclaurin coefficients $\left|a_{2}\right|$ and $\left|a_{3}\right|$ (for details see [2]).

The study of operators plays an important role in the geometric function theory and its related fields.Many differential and integral operators can be 
written in terms of convolution of certain analytic functions. It is observed that this formalism brings an ease in further mathematical exploration and also helps to understand the geometric properties of such operators better. We recall a sufficiently adequate special case of the generalized convolution operator $\mathcal{I}_{a, b, c}$, due to Hohlov [4] in terms of the Hadamard product (or convolution).

The convolution or Hadamard product of two functions $f, h \in \mathcal{A}$ is denoted by $f * h$ and is defined as

$$
(f * h)(z)=z+\sum_{n=2}^{\infty} a_{n} b_{n} z^{n},
$$

where $f(z)$ is given by (1.1) and $h(z)=z+\sum_{n=2}^{\infty} b_{n} z^{n}$.

For the complex parameters $a, b$ and $c$ with $c \neq 0,-1,-2,-3, \cdots$, the Gaussian hypergeometric function ${ }_{2} F_{1}(a, b, c ; z)$ is defined as

$$
\begin{aligned}
{ }_{2} F_{1}(a, b, c ; z) & =\sum_{n=0}^{\infty} \frac{(a)_{n}(b)_{n}}{(c)_{n} n !} z^{n}, \\
& =1+\sum_{n=2}^{\infty} \frac{(a)_{n-1}(b)_{n-1}}{(c)_{n-1}(n-1) !} z^{n-1}, \quad z \in \mathbb{U},
\end{aligned}
$$

where $(\alpha)_{n}$ is the Pochhammer symbol (or the shifted factorial) defined as

$$
(\alpha)_{n}=\frac{\Gamma(\alpha+n)}{\Gamma(\alpha)}= \begin{cases}1, & \text { if } n=0 \\ \alpha(\alpha+1)(\alpha+2) \cdots(\alpha+n-1), & \text { if } n \neq 0 .\end{cases}
$$

By using the Gaussian hypergeometric function given by (1.5), Hohlov [4] introduced a generalized convolution operator $\mathcal{I}_{a, b, c}$ as

$$
\begin{aligned}
\mathcal{I}_{a, b ; c} f(z) & =z{ }_{2} F_{1}(a, b, c ; z) * f(z), \\
& =z+\sum_{n=2}^{\infty} \Gamma_{n} a_{n} z^{n}, z \in \mathbb{U},
\end{aligned}
$$

where

$$
\Gamma_{n}=\frac{(a)_{n-1}(b)_{n-1}}{(c)_{n-1}(n-1) !}
$$

and discussed some interesting geometrical properties exhibited by this operator. The three parameter family of operators $\mathcal{I}_{a, b ; c}$ contains as a special cases most of the known linear integral or differential operators. In particular, if 
$b=1$ in (1.7), then $\mathcal{I}_{a, 1 ; c}$ reduces to the Carlson-Shaffer operator a special case of Hohlov operator. Similarly it is straightforward to show that Hohlov operator is also a generalization of Ruscheweyh and Bernardi operators.

The object of the present paper is to introduce two new subclasses of the function class $\Sigma$ and find estimate on the coefficients $\left|a_{2}\right|$ and $\left|a_{3}\right|$ for functions in these new subclasses of the function class $\Sigma$ employing the techniques used earlier by Srivastava et al. [11] and others (see[3, 8, 10, 12, 13]).

Definition 1.1. For $0<\alpha \leq 1 ; 0 \leq \lambda \leq 1$, a function $f(z)$ given by (1.1) is said to be in the class $\mathcal{P}_{\Sigma}^{a, b ; c}(\alpha, \lambda)$ if the following conditions are satisfied:

$$
f \in \Sigma,\left|\arg \left(\frac{z\left(\mathcal{I}_{a, b ; c} f(z)\right)^{\prime}+\lambda z^{2}\left(\mathcal{I}_{a, b ; c} f(z)\right)^{\prime \prime}}{(1-\lambda) \mathcal{I}_{a, b ; c} f(z)+\lambda z\left(\mathcal{I}_{a, b ; c} f(z)\right)^{\prime}}\right)\right|<\frac{\alpha \pi}{2},
$$

and

$$
\left|\arg \left(\frac{w\left(\mathcal{I}_{a, b ; c} g(w)\right)^{\prime}+\lambda w^{2}\left(\mathcal{I}_{a, b ; c} g(w)\right)^{\prime \prime}}{(1-\lambda) \mathcal{I}_{a, b ; c} g(w)+\lambda w\left(\mathcal{I}_{a, b ; c} g(w)\right)^{\prime}}\right)\right|<\frac{\alpha \pi}{2},
$$

where $z, w \in \mathbb{U}$ and the function $g$ is given by (1.3).

Definition 1.2. For $0 \leq \beta<1 ; 0 \leq \lambda \leq 1$, a function $f(z)$ given by (1.1) is said to be in the class $\mathcal{Q}_{\Sigma}^{a, b ; c}(\beta, \lambda)$ if the following conditions are satisfied:

$$
f \in \Sigma, \Re\left(\frac{z\left(\mathcal{I}_{a, b ; c} f(z)\right)^{\prime}+\lambda z^{2}\left(\mathcal{I}_{a, b ; c} f(z)\right)^{\prime \prime}}{(1-\lambda) \mathcal{I}_{a, b ; c} f(z)+\lambda z\left(\mathcal{I}_{a, b ; c} f(z)\right)^{\prime}}\right)>\beta,
$$

and

$$
\Re\left(\frac{w\left(\mathcal{I}_{a, b ; c} g(w)\right)^{\prime}+\lambda w^{2}\left(\mathcal{I}_{a, b ; c} g(w)\right)^{\prime \prime}}{(1-\lambda) \mathcal{I}_{a, b ; c} g(w)+\lambda w\left(\mathcal{I}_{a, b ; c} g(w)\right)^{\prime}}\right)>\beta,
$$

where $z, w \in \mathbb{U}$ and the function $g$ is given by (1.3).

Example 1. For $\lambda=0$, and $0<\alpha \leq 1$ we let $\mathcal{P}_{\Sigma}^{a, b ; c}(\alpha, 0)=\mathcal{S}_{\Sigma}^{a, b ; c}(\alpha)$ and $\mathcal{Q}_{\Sigma}^{a, b ; c}(\beta, 0)=\mathcal{S}_{\Sigma}^{a, b ; c}(\beta)$ be the subclasses of $\Sigma$ consisting functions of the form (1.1) satisfying the condition

$$
\left|\arg \left(\frac{z\left(\mathcal{I}_{a, b ; c} f(z)\right)^{\prime}}{\mathcal{I}_{a, b ; c} f(z)}\right)\right|<\frac{\alpha \pi}{2} ;\left|\arg \left(\frac{w\left(\mathcal{I}_{a, b ; c} g(w)\right)^{\prime}}{\mathcal{I}_{a, b ; c} g(w)}\right)\right|<\frac{\alpha \pi}{2}
$$

and

$$
\Re\left(\frac{z\left(\mathcal{I}_{a, b ; c} f(z)\right)^{\prime}}{\mathcal{I}_{a, b ; c} f(z)}\right)>\beta ; \Re\left(\frac{w\left(\mathcal{I}_{a, b ; c} g(w)\right)^{\prime}}{\mathcal{I}_{a, b ; c} g(w)}\right)>\beta
$$

respectively,where the function $g$ is given by (1.3)and $z, w \in \mathbb{U}$. 
Example 2. For $\lambda=1$ we let $\mathcal{P}_{\Sigma}^{a, b ; c}(\alpha, 1)=\mathcal{K}_{\Sigma}^{a, b ; c}(\alpha) \quad$ and $\mathcal{Q}_{\Sigma}^{a, b ; c}(\beta, 1)=\mathcal{K}_{\Sigma}^{a, b ; c}(\beta)$ be the subclasses of $\Sigma$ satisfying the condition that

$$
\left|\arg \left(1+\frac{z\left(\mathcal{I}_{a, b ; c} f(z)\right)^{\prime \prime}}{\left(\mathcal{I}_{a, b ; c} f(z)\right)^{\prime}}\right)\right|<\frac{\alpha \pi}{2} ;\left|\arg \left(1+\frac{w\left(\mathcal{I}_{a, b ; c} g(w)\right)^{\prime \prime}}{\left(\mathcal{I}_{a, b ; c} g(w)\right)^{\prime}}\right)\right|<\frac{\alpha \pi}{2}
$$

and

$$
\Re\left(1+\frac{z\left(\mathcal{I}_{a, b ; c} f(z)\right)^{\prime \prime}}{\left(\mathcal{I}_{a, b ; c} f(z)\right)^{\prime}}\right)>\beta ; \Re\left(1+\frac{w\left(\mathcal{I}_{a, b ; c} g(w)\right)^{\prime \prime}}{\left(\mathcal{I}_{a, b ; c} g(w)\right)^{\prime}}\right)>\beta
$$

respectively,where the function $g$ is given by (1.3)and $z, w \in \mathbb{U}$.

Further it is of interest to note that by taking $a=b$ and $c=1$ in the Definitions 1.1and 1.2 we deduce the new subclasses of $\Sigma$ defined and discussed by authors in[6]

In order to derive our main results, we have to recall here the following lemma [9].

Lemma 1.3. If $h \in \wp$ then $\left|c_{k}\right| \leq 2$ for each $k$, where $\wp$ is the family of all functions $h$ analytic in $\mathbb{U}$ for which $\Re\{h(z)\}>0$, where $h(z)=1+c_{1} z+c_{2} z^{2}+\ldots$ for $z \in \mathbb{U}$.

In the following section, we obtain the estimates on the coefficients $\left|a_{2}\right|$ and $\left|a_{3}\right|$ for $f \in \mathcal{P}_{\Sigma}^{a, b ; c}(\alpha, \lambda)$. and $f \in \mathcal{Q}_{\Sigma}^{a, b ; c}(\beta, \lambda)$.

\section{Coefficient Bounds for the Function Class $\mathcal{P}_{\Sigma}^{a, b ; c}(\alpha, \lambda)$}

Theorem 2.1. Let $f(z)$ given by (1.1) be in the class $\mathcal{P}_{\Sigma}^{a, b ; c}(\alpha, \lambda), 0<$ $\alpha \leq 1$ and $0 \leq \lambda \leq 1$. Then

$$
\left|a_{2}\right| \leq \frac{2 \alpha}{\sqrt{4 \alpha(1+2 \lambda) \Gamma_{3}+(1-3 \alpha)(1+\lambda)^{2} \Gamma_{2}^{2}}}
$$

and

$$
\left|a_{3}\right| \leq \frac{4 \alpha^{2}}{(1+\lambda)^{2} \Gamma_{2}^{2}}+\frac{\alpha}{(1+2 \lambda) \Gamma_{3}} .
$$

Proof. It follows from (1.9) and (1.10) that

$$
\frac{z\left(\mathcal{I}_{a, b ; c} f(z)\right)^{\prime}+\lambda z^{2}\left(\mathcal{I}_{a, b ; c} f(z)\right)^{\prime \prime}}{(1-\lambda) \mathcal{I}_{a, b ; c} f(z)+\lambda z\left(\mathcal{I}_{a, b ; c} f(z)\right)^{\prime}}=[p(z)]^{\alpha}
$$


and

$$
\frac{w\left(\mathcal{I}_{a, b ; c} g(w)\right)^{\prime}+\lambda w^{2}\left(\mathcal{I}_{a, b ; c} g(w)\right)^{\prime \prime}}{(1-\lambda) \mathcal{I}_{a, b ; c} g(w)+\lambda w\left(\mathcal{I}_{a, b ; c} g(w)\right)^{\prime}}=[q(w)]^{\alpha},
$$

where $p(z)$ and $q(w)$ in $\wp$ and have the forms

$$
p(z)=1+p_{1} z+p_{2} z^{2}+\ldots
$$

and

$$
q(w)=1+q_{1} w+q_{2} w^{2}+\ldots
$$

Now, equating the coefficients in (2.3) and (2.4), we get

$$
\begin{aligned}
(1+\lambda) \Gamma_{2} a_{2} & =\alpha p_{1} \\
-(1+\lambda)^{2} \Gamma_{2}^{2} a_{2}^{2}+2(1+2 \lambda) \Gamma_{3} a_{3} & =\frac{1}{2}\left[\alpha(\alpha-1) p_{1}^{2}+2 \alpha p_{2}\right] \\
-(1+\lambda) \Gamma_{2} a_{2} & =\alpha q_{1}
\end{aligned}
$$

and

$$
\left[4(1+2 \lambda) \Gamma_{3}-(1+\lambda)^{2} \Gamma_{2}^{2}\right] a_{2}^{2}-2(1+2 \lambda) \Gamma_{3} a_{3}=\frac{1}{2}\left[\alpha(\alpha-1) q_{1}^{2}+2 \alpha q_{2}\right] .
$$

From (2.7) and (2.9), we get

$$
p_{1}=-q_{1}
$$

and

$$
2(1+\lambda)^{2} \Gamma_{2}^{2} a_{2}^{2}=\alpha^{2}\left(p_{1}^{2}+q_{1}^{2}\right) .
$$

From (2.8), (2.10) and (2.12), we obtain

$$
a_{2}^{2}=\frac{\alpha^{2}\left(p_{2}+q_{2}\right)}{4 \alpha(1+2 \lambda) \Gamma_{3}+(1-3 \lambda)(1+\lambda)^{2} \Gamma_{2}^{2}} .
$$

Applying Lemma 1.3 for the coefficients $p_{2}$ and $q_{2}$, we immediately have

$$
\left|a_{2}\right| \leq \frac{2 \alpha}{\sqrt{4 \alpha(1+2 \lambda) \Gamma_{3}+(1-3 \lambda)(1+\lambda)^{2} \Gamma_{2}^{2}}}
$$

This gives the bound on $\left|a_{2}\right|$ as asserted in (2.1).

Next, in order to find the bound on $\left|a_{3}\right|$, by subtracting (2.10) from (2.8), we get

$$
4(1+2 \lambda) \Gamma_{3} a_{3}-4(1+2 \lambda) \Gamma_{3} a_{2}^{2}=\alpha\left(p_{2}-q_{2}\right)+\frac{\alpha(\alpha-1)}{2}\left(p_{1}^{2}-q_{1}^{2}\right) .
$$


It follows from (2.11), (2.12) and (2.13) that

$$
a_{3}=\frac{\alpha\left(p_{2}-q_{2}\right)}{4(1+2 \lambda) \Gamma_{3}}+\frac{\alpha^{2}\left(p_{1}^{2}+q_{1}^{2}\right)}{2(1+\lambda)^{2} \Gamma_{2}^{2}} .
$$

Applying Lemma 1.3 once again for the coefficients $p_{1}, p_{2}, q_{1}$ and $q_{2}$, we readily get

$$
\left|a_{3}\right| \leq \frac{4 \alpha^{2}}{(1+\lambda)^{2} \Gamma_{2}^{2}}+\frac{\alpha}{(1+2 \lambda) \Gamma_{3}} .
$$

This completes the proof of Theorem 2.1.

\section{Coefficient Bounds for the Function Class $\mathcal{Q}_{\Sigma}^{a, b ; c}(\beta, \lambda)$}

Theorem 3.1. Let $f(z)$ given by (1.1) be in the class $\mathcal{Q}_{\Sigma}^{a, b ; c}(\beta, \lambda), 0 \leq$ $\beta<1$ and $0 \leq \lambda \leq 1$. Then

$$
\left|a_{2}\right| \leq \sqrt{\frac{2(1-\beta)}{2(1+2 \lambda) \Gamma_{3}-(1+\lambda)^{2} \Gamma_{2}^{2}}}
$$

and

$$
\left|a_{3}\right| \leq \frac{4(1-\beta)^{2}}{(1+\lambda)^{2} \Gamma_{2}^{2}}+\frac{(1-\beta)}{(1+2 \lambda) \Gamma_{3}} .
$$

Proof. It follows from (1.11) and (1.12) that there exists $p, q \in \wp$ such that

$$
\frac{z\left(\mathcal{I}_{a, b ; c} f(z)\right)^{\prime}+\lambda z^{2}\left(\mathcal{I}_{a, b ; c} f(z)\right)^{\prime \prime}}{(1-\lambda) \mathcal{I}_{a, b ; c} f(z)+\lambda z\left(\mathcal{I}_{a, b ; c} f(z)\right)^{\prime}}=\beta+(1-\beta) p(z)
$$

and

$$
\frac{w\left(\mathcal{I}_{a, b ; c} g(w)\right)^{\prime}+\lambda w^{2}\left(\mathcal{I}_{a, b ; c} g(w)\right)^{\prime \prime}}{(1-\lambda) \mathcal{I}_{a, b ; c} g(w)+\lambda w\left(\mathcal{I}_{a, b ; c} g(w)\right)^{\prime}}=\beta+(1-\beta) q(w),
$$

where $p(z)$ and $q(w)$ have the forms (2.5) and (2.6), respectively. Equating coefficients in (3.3) and (3.4), we get

$$
\begin{gathered}
(1+\lambda) \Gamma_{2} a_{2}=(1-\beta) p_{1} \\
-(1+\lambda)^{2} \Gamma_{2}^{2} a_{2}^{2}+2(1+2 \lambda) \Gamma_{3} a_{3}=(1-\beta) p_{2} \\
-(1+\lambda) \Gamma_{2} a_{2}=(1-\beta) q_{1}
\end{gathered}
$$


and

$$
\left[4(1+2 \lambda) \Gamma_{3}-(1+\lambda)^{2} \Gamma_{2}^{2}\right] a_{2}^{2}-2(1+2 \lambda) \Gamma_{3} a_{3}=(1-\beta) q_{2} .
$$

From (3.5) and (3.7), we get

$$
p_{1}=-q_{1}
$$

and

$$
2(1+\lambda)^{2} \Gamma_{2}^{2} a_{2}^{2}=(1-\beta)^{2}\left(p_{1}^{2}+q_{1}^{2}\right) .
$$

Also, from (3.6)and (3.8), we obtain

$$
a_{2}^{2}=\frac{(1-\beta)\left(p_{2}+q_{2}\right)}{2\left[2(1+2 \lambda) \Gamma_{3}-(1+\lambda)^{2} \Gamma_{2}^{2}\right]} .
$$

Applying Lemma 1.3 for the coefficients $p_{2}$ and $q_{2}$, we immediately have

$$
\left|a_{2}\right| \leq \sqrt{\frac{2(1-\beta)}{2(1+2 \lambda) \Gamma_{3}-(1+\lambda)^{2} \Gamma_{2}^{2}}} .
$$

This gives the bound on $\left|a_{2}\right|$ as asserted in (3.1).

Next, in order to find the bound on $\left|a_{3}\right|$, by subtracting (3.8) from (3.6), we get

$$
4(1+2 \lambda) \Gamma_{3} a_{3}-4(1+2 \lambda) \Gamma_{3} a_{2}^{2}=(1-\beta)\left(p_{2}-q_{2}\right) .
$$

It follows from (3.10) and (3.11) that

$$
a_{3}=\frac{(1-\beta)\left(p_{2}-q_{2}\right)}{4(1+2 \lambda) \Gamma_{3}}+\frac{(1-\beta)^{2}\left(p_{1}^{2}+q_{1}^{2}\right)}{2(1+\lambda)^{2} \Gamma_{2}^{2}} .
$$

Applying Lemma 1.3 once again for the coefficients $p_{1}, p_{2}, q_{1}$ and $q_{2}$, we readily get

$$
\left|a_{3}\right| \leq \frac{4(1-\beta)^{2}}{(1+\lambda)^{2} \Gamma_{2}^{2}}+\frac{1-\beta}{(1+2 \lambda) \Gamma_{3}} .
$$

This completes the proof of Theorem 3.1.

\section{Concluding Remarks}

Various other interesting corollaries and consequences of our main results (which are asserted by Theorems 2.1 3.1and above) can be derived similarly for the new classes defined in Example 1 and Example2.Further by taking $a=b$ and $c=1$ in Theorems 2.1 3.1we get the results discussed by authors in [6]. 


\section{References}

[1] D.A. Brannan, J.G. Clunie (Eds.), Aspects of Contemporary Complex Analysis (Proceedings of the NATO Advanced Study Institute held at the University of Durham, Durham; July 1-20, 1979), Academic Press, New York and London, 1980.

[2] D.A. Brannan, T.S. Taha, On some classes of bi-univalent functions, Studia Univ. Babe-Bolyai Math. 31 (2) (1986) 70-77.

[3] B.A.Frasin and M.K.Aouf, New subclasses of bi-univalent functions, Appl. Math. Lr., 24(2011), 1569-1573.

[4] Yu. E. Hohlov, Hadamard convolutions, hypergeometric functions and linear operators in the class of univalent functions, Dokl. Akad. Nauk Ukrain. SSR Ser. A 1984, no. 7, 25-27.

[5] M. Lewin, On a coefficient problem for bi-univalent functions, Proc. Amer. Math. Soc. 18 (1967) 63-68.

[6] G. Murugusundaramoorthy,C.selvaraj and O.S.Babu, Coefficient estimates for Pascu-type subclasses of bi-univalent functions based on subordination,International Journal of Nonlinear Science(IJNS)(to appear in 2015).

[7] E. Netanyahu, The minimal distance of the image boundary from the origin and the second coefficient of a univalent function in $|z|<1$, Arch. Rational Mech. Anal. 32 (1969) 100-112.

[8] T.Panigarhi and G. Murugusundaramoorthy, Coefficient bounds for Biunivalent functions analytic functions associated with Hohlov operator, Proc. Jangjeon Math. Soc,16 (1) (2013) 91-100

[9] Ch. Pommerenke, Univalent Functions, Vandenhoeck and Rupercht, Göttingen, 1975.

[10] H. M. Srivastava, G. Murugusundaramoorthy and N. Magesh,, Certain subclasses of bi-univalent functions associated with the Hohlov operator ,Global Journal of Mathematical Analysis, 1 (2) (2013) 67-73.

[11] H.M.Srivastava, A.K.Mishra and P.Gochhayat, Certain subclasses of analytic and bi-univalent functions, Appl. Math. Lr., 23(2010), 1188-1192. 
[12] Q.-H. Xu, Y.-C. Gui and H. M. Srivastava, Coefficinet estimates for a certain subclass of analytic and bi-univalent functions, Appl. Math. Lett. 25(2012), 990-994.

[13] Q.-H.Xu, H.-G.Xiao and H. M. Srivastava, A certain general subclass of analytic and bi-univalent functions and associated coefficient estimate problems, Appl. Math. Comput. 218(2012), 11461-11465. 